\title{
CUIDADOS PALIATIVOS NO PROCESSO DE SENESCÊNCIA: REVISÃO SISTEMÁTICA DE LITERATURA
}

\author{
1, ${ }^{*}$ Kalile dos Anjos Souza, ${ }^{2}$ Mauro Fernandes Teles, ${ }^{3}$ Diêgo Andrade de Oliveira, ${ }^{4}$ Mariane Costa \\ Santos de Tavares, ${ }^{5}$ Rosy Aline Lopes de Oliveira, ${ }^{5}$ Ana Carolina da Silva Soares Martins, \\ ${ }^{5}$ Vanessa Ramalho Correia, ${ }^{5}$ Tamires Batista Pedreira, ${ }^{6}$ Daniely Sampaio Ribeiro, ${ }^{5}$ Melissa \\ Menezes Tolentino, ${ }^{7}$ Caroline Braga Palacio, ${ }^{5}$ Ananda Mendes Antunes and \\ ${ }^{5}$ Ana Maria Lima Tigre
}

${ }^{1}$ Autora Correspondente. Discente do curso de Graduação em Medicina. Faculdades Santo Agostinho de Vitória da Conquista - FASAVIC, Vitória da Conquista - BA. Brasil. ${ }^{2}$ Farmacêutico. Mestre em Saúde Pública pela Escola

Nacional de Saúde Pública Sergio Arouca ENSP/FIOCRUZ. Docente do curso de Graduação em Medicina.

Faculdades Santo Agostinho de Vitória da Conquista - FASAVIC, Vitória da Conquista - BA. Brasil.

${ }^{3}$ Biólogo. Mestre em Biologia e Biotecnologia de Microrganismos. Docente do curso de Graduação em Medicina.

Faculdades Santo Agostinho de Vitória da Conquista - FASAVIC, Vitória da Conquista - BA. Brasil.

${ }^{4}$ Fisioterapeuta. Especialista em Fisioterapia Respiratória e Terapia Intensiva pelas Faculdades Integradas

Pitágoras, Pós -Graduada em Deficiências Múltiplas e Sensoriais. Discente do Curso de Graduação em Medicina

Faculdades Santo Agostinhode Vitória da Conquista - FASAVIC, Vitória da Conquista - BA. Brasil.

${ }^{5}$ Discente do curso de Graduação em Medicina. Faculdades Santo Agostinho de Vitória da Conquista - FASAVIC, Vitória da Conquista - BA. Brasil. ${ }^{6}$ Discente do curso de Graduação em Medicina. Faculdades Santo Agostinho de

Itabuna - FASA, Itabuna - BA. Brasil. ${ }^{7}$ Médica. DSEI Alto Rio Solimões, Pólo Base Belém do Solimões

\begin{tabular}{l} 
ARTICLE INFO \\
\hline Article History: \\
Received $06^{\text {th }}$ January, 2021 \\
Received in revised form \\
$19^{\text {th }}$ February, 2021 \\
Accepted $11^{\text {th }}$ March, 2021 \\
Published online $22^{\text {th }}$ April, 2021 \\
\hline
\end{tabular}

Key Words:

Cuidados Paliativos. Atenção à Saúde do Idoso. Serviços de Saúde para Idosos.

*Corresponding author: Kalile dos Anjos Souza

\begin{abstract}
O envelhecimento populacional é um fenômeno global, que exige da sociedade, poder público e serviços assistenciais uma reestruturação de processos, legislação e posturas. Em tal contexto, verifica-se o crescimento acelerado de estudos e serviços voltados aos cuidados paliativos no processo de senescência. O objetivo do estudo foi conhecer o significado de cuidados paliativos ao idoso na percepção do médico e equipe multidisciplinar, bem como analisar as comorbidades relacionadas ao processo de senescência. Sob o aspecto metodológico o estudo caracteriza-se como uma revisão sistemática de literatura, realizada nas bases de dados vinculadas à Medical Literature Analysis and Retrieval System Online (MEDLINE), e Literatura Latino-americana e do Caribe em Ciências da Saúde (LILACS). Foram utilizados os descritores em ciências da saúde (DECS): Cuidados Paliativos, Atenção à Saúde do Idoso, Envelhecimento. Foram selecionados para análise e discussão quatorze estudos que abordavam a assistência em cuidados paliativos ao idoso. Verifica-se grande carência na estruturação de serviços, bem como no preparo profissional para um cuidado adequado. $\mathrm{O}$ desrespeito à autonomia do idoso, e ausência de comunicação efetiva entre equipe de saúde e paciente reflete ainda a predominância de um modelo biomédico latente, em que não há individualidade do cuidado, e sobressai o paternalismo médicoassistencial, em detrimento ao respeito da vontade e valores do paciente.
\end{abstract}

Copyright (C) 2021, Kalile dos Anjos Souza et al. This is an open access article distributed under the Creative Commons Attribution License, which permits unrestricted use, distribution, and reproduction in any medium, provided the original work is properly cited. 


\section{INTRODUCTION}

Envelhecer é um processo contínuo inerente à condição humana. Compreende todo um conjunto de mudanças orgânicas, psíquicas e sociais de natureza progressiva que é influenciada pela genética e por hábitos e comportamentos que o indivíduo assume durante sua vida. $\mathrm{O}$ envelhecimento é marcado pela redução das funções orgânicas e cognitivas, maior predisposição para o desenvolvimento de doenças degenerativas crônicas e perda de independência nas atividades de vida diária. Existe um declínio das funções fisiológicas nesse processo contínuo, que é a senescência(Tavares \& Lima, 2017). Cuidados Paliativos (CPs) são definidos pela Organização Mundial da Saúde (OMS) como a assistência prestada ao paciente terminal com o intuito de reduzir o seu sofrimento, bem como de seus familiares, buscando proporcionar a melhor qualidade de vida possível à estes. Além disso, preconiza-se trabalhar com pacientes e família a noção de que a morte é parte integrante da vida, e este ciclo precisa ser aceito como natural. Ainda de acordo com a OMS o paciente terminal precisa ser assistido por uma equipe multidisciplinar, abordando sintomas físicos, psíquicos, sociais e espirituais(WHO, 2002). Frequentemente o adoecimento gera nas pessoas afetadas, nos profissionais e familiares um intenso desejo de que durante esse processo se encontrem alternativas de tratamento e cura. A resposta adequada ao tratamento confirma o êxito de uma batalha contra um inimigo biológico desencadeador de pesados dissabores à vida do ser humano. Frente à impossibilidade da cura, busca-se então a oferta de cuidados paliativos. Tais cuidados aqui entendidos como aqueles que proporcionam, ao paciente cuja doença não é mais responsiva a tratamento curativo,o conforto, o alívio da dor e do sofrimento, bem como, melhora na qualidade de vida nos aspectos físico, psicológico, social e espiritual, com ênfase no amparo à família durante todo o processo de doença, morte e luto(Academia Nacional de Cuidados Paliativos, 2009). Emprega-se o conceito cuidados paliativos para designar a ação de uma equipe multiprofissional a pacientes fora de possibilidades terapêuticas de cura. $\mathrm{O}$ termo paliativo é originado do latim palliun que significa manto, proteção, ou seja, proteger aqueles aos quais a medicina curativa já não mais acolhe(Correia, Nascimento, Santos, Santos, \& Oliveira, 2017).

Envelhecer é um processo natural que implica em mudanças graduais e inevitáveis relacionadas à idade e sucede a despeito de um indivíduo gozar de boa saúde e ter um estilo de vida ativo e saudável. No ser humano, esse fenômeno progressivo, além de desencadear o desgaste orgânico, provoca alterações nos aspectos culturais, sociais e emocionais que contribuem para que se instale em diferentes idades cronológicas(Ciosak, et al., 2011). A Organização Mundial de Saúde (OMS) considera o envelhecer como: "um processo sequencial, individual, cumulativo, irreversível, universal, não patológico de deterioração de um organismo maduro, próprio a todos os membros de uma espécie, de maneira que o tempo o torne menos capaz de fazer frente ao estresse do meio ambiente e, portanto, aumente sua possibilidade de morte".

Ainda para a OMS, o limite de idade entre o indivíduo adulto e o idoso é 65 anos em nações desenvolvidas e 60 nos países emergentes(Farias \& Santos, 2012). Logo, o envelhecimento populacional é uma consequência do nível de desenvolvimento de uma nação. $\mathrm{O}$ aumento proporcional de indivíduos idosos, adicionado ao declínio das taxas de fecundidade e ao desenvolvimento tecnológico e terapêutico no tratamento de doenças, especialmente as crônicas, influenciam a tendência de alteração da estrutura etária da população, inclusive no Brasil, com o consequente aumento do contingente de indivíduos com mais de 60 anos, resultado do envelhecimento populacional que ocorreu em um curto período, trazendo importante impacto para o sistema de saúde(Miranda, Mendes, \& Silva, 2016). Neste sentido, sabendo-se ser o crescimento demográfico do extrato populacional de idoso uma realidade em todo o mundo, considera-se relevante a preocupação com esse grupo, porque, em geral, são pessoas acometidas por doenças crônicas não transmissíveis que as levam a condições de cronicidade.
Em tal conjuntura, o presente estudo teve como objetivo conhecer o significado de cuidados paliativos ao idoso na percepção do médico e equipe multidisciplinar, bem como analisar as comorbidades relacionadas ao processo de senescência.

\section{METODOLÓGICOS}

O estudo realizado caracteriza-se como uma revisão sistemática de literatura, realizada nas bases de dados vinculadas à Medical Literature Analysis and Retrieval System Online (MEDLINE), e Literatura Latino-americana e do Caribe em Ciências da Saúde (LILACS). Foram utilizados os descritores em ciências da saúde (DECS): Cuidados Paliativos, Atenção à Saúde do Idoso, Envelhecimento. Considerou-se estudos publicados nos últimos cinco anos, que abordavam a temática de cuidados paliativos na atenção à saúde do idoso. A triagem dos estudos obedeceu os pressupostos da metodologia PRISMA. A análise dos dados se deu por técnica de análise de conteúdo e abordagem qualitativa das informações coletadas.

\section{RESULTADOS E DISCUSSÃO}

No fluxograma 1 está apresentada a triagem dos estudos e o processo de seleção destes.

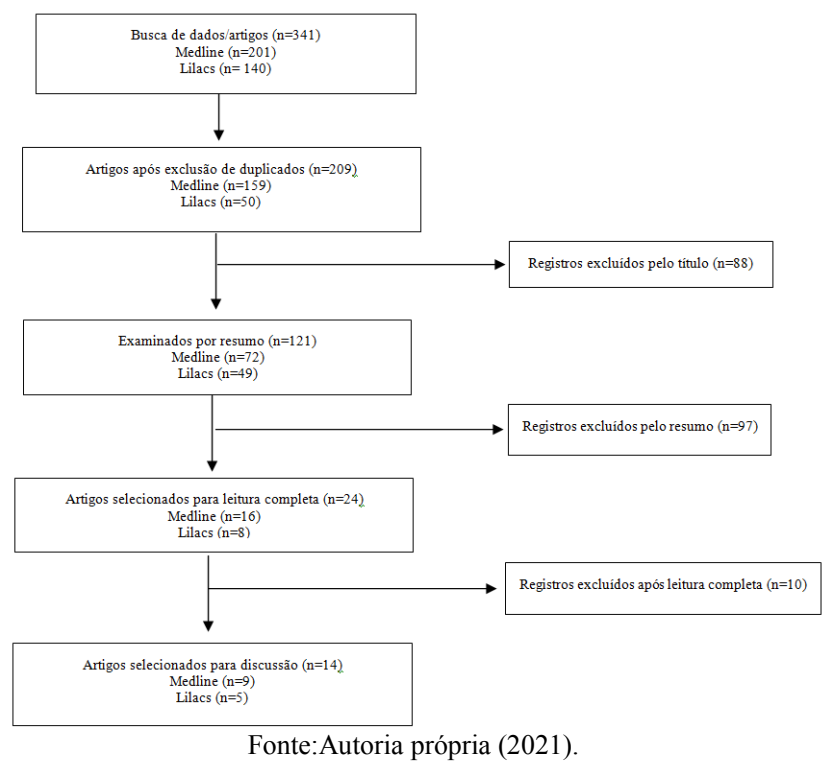

Fluxograma 1. Triagem e seleção de estudos conforme metodologia PRISMA

Processo de envelhecimento e indicação do cuidado paliativo: $\mathrm{O}$ envelhecimento é algo que ocorre paulatinamente, uma vez que o indivíduo começa esse processo ao nascer. Entretanto, considerando os aspectos biofuncionais, o mesmo tem início na segunda década de vida, embora de forma imperceptível. No final da terceira década surgem as primeiras alterações funcionais e estruturais e, a partir da quarta, há uma perda de aproximadamente $1 \%$ da função/ano, nos diferentes sistemas orgânicos (Liposcki, 2016). O envelhecer natural está ligado à capacidade de adaptação do indivíduo aos rigores e às agressões do meio ambiente. Assim, cada sujeito envelhece a seu modo, dependendo de variáveis como: sexo, origem, lugar em que vive, tamanho da família, aptidões para a vida e as experiências vivenciadas. A exposição ao estresse ou ao tabagismo, a falta de exercícios físicos ou a nutrição inadequada são outros fatores que contribuem para determinar a qualidade do envelhecimento (Abenante, 2021). O envelhecimento e a doença não podem ser tratados como fatores intimamente dependentes ou interligados, porém existe maior vulnerabilidade para adoecer, ou seja, uma 
predisposição à doença. A saúde e a qualidade de vida dos idosos, mais que em outros grupos etários, sofrem influência de múltiplos fatores: físicos, psicológicos, sociais e culturais, de tal forma que avaliar e promover a saúde do idoso significa considerar variáveis de distintos campos do saber, numa atuação interdisciplinar e multidimensional (Liposcki, 2016). A assistência ao idoso deve prezar pela manutenção da qualidade de vida, considerando os processos de perdas próprias do envelhecimento e as possibilidades de prevenção, manutenção e reabilitação do seu estado de saúde. Considerando que o processo saúde-doença é um fenômeno complexo, socialmente determinado e modulado por condicionantes biológicos, psicológicos, culturais, econômicos e políticos, as necessidades de saúde dos idosos referem-se à múltiplas dimensões do real e dizem respeito à singularidade dos fenômenos de saúde ou doença que afetam os indivíduos e suas famílias (Ribeiro, 2017). De acordo com a Organização Mundial da Saúde os cuidados paliativos buscam aliviar o sofrimento da pessoa em condição de terminalidade, garantindo conforto físico, psíquico, social e espiritual. Dados recentes apontam que em países de média e baixa renda menos de $10 \%$ dos pacientes que precisam de cuidados paliativos de fato os recebem, evidenciando uma grande demanda e lacuna assistencial (World Health Organization - WHO, 2017). Este fato também foi evidenciado por Gomes \& Othero (2016) que apontam a existência de um grande paradoxo. Se por um lado os avanços tecnológicos e científicos promovem a redução de taxa de mortalidade e envelhecimento populacional, por outro, idosos acometidos por doenças crônico-degenerativas graves não são beneficiados por recursos modernos no momento do morrer. Grande parte dos profissionais, ao evidenciarem a inexistência da possibilidade de cura deixam de assistir o idoso adequadamente, não havendo a instituição adequada do cuidado paliativo (Gomes \& Othero, 2016).

Conforme a literatura há uma dificuldade em estabelecer critérios para instituição de cuidados paliativos ao idoso. Um dos instrumentos propostos foi a escala Palliative CareScreening Tool (PCST) desenvolvida pelo Center to Advance PalliativeCare (CAPC). Tal instrumento utiliza os critérios de doença de base e quadros mórbidos associados, condição funcional, bem como condições pessoais do paciente. Um ponto importante destacado é que tal instrumento não leva em consideração aspectos de religiosidade e espiritualidade dos idosos (Clara, Silva, Alves, \& Coelho, 2019). Em outro estudo buscou-se identificar características laboratoriais e clínicas associadas à indicação de cuidados paliativos para idosos gravemente enfermos. Foram incluídos 572 prontuários de idosos gravemente enfermos, sendo que $27 \%$ foi considerado elegível para cuidados paliativos. Os diagnósticos mais comuns foram estado de demência avançada $(45 \%)$, neoplasias $(38 \%)$, insuficiência cardíaca congestiva - ICC (25\%), insuficiência renal grave (24\%), doença pulmonar obstrutiva crônica - DPOC $(8 \%)$ e cirrose $(4 \%)$. Os preditores independentes para indicação de cuidados exclusivamente paliativos foram: sexo masculino, câncer, demência avançada e níveis de albumina. Dentre as complicações mais evidenciadas entre pacientes idosos em cuidados paliativos foram destacadas: delírio, infecções e úlceras por pressão (Arcanjo, Saporetti, Curiati, Jacob-Filho, \& Silva, 2018).

Prática de Cuidados paliativos no idoso: No Brasil o processo de cuidados paliativos é ancorado pela Política Nacional de Humanização da Atenção e da Gestão na Saúde (PNH), que busca a humanização do cuidado ao paciente em todos os ciclos de vida, incluindo a terminalidade desta. Infelizmente os estudos apontam que o envelhecimento ainda está intimamente associado à vivência de "anos de sofrimento", prolongadas internações hospitalares, baixa qualidade de vida e carência afetiva, social, e assistencial (Alves, Cunha, Santos, \& Melo, 2019). Os cuidados paliativos no ambiente domiciliar na realidade brasileira são descritos como uma situação necessária, embora mal estruturada e angustiante para profissionais, pacientes e familiares. Há elevada carência de recursos, preparo técnico e rede de suporte no contexto do Sistema Único de Saúde (SUS). Os próprios gestores da Atenção Primária por vezes incluem os idosos em cuidados paliativos de forma superficial e incompleta, seja por sobrecarga nos serviços, ou mesmo por ausência de planejamento para ações resolutivas de cuidados paliativos (Marques
\& Bulgarelli, 2020). Há grande discussão da literatura sobre o local apropriado para estruturação dos cuidados paliativos. Embora grande parte destes idosos acabe tendo seus últimos dias de vida em Unidades de Terapia Intensiva (UTI) verifica-se consenso entre os autores que este não seria o espaço "mais indicado" (Queiroz, et al., 2018). Em pacientes domiciliados a Atenção Primária à Saúde (APS) poderia exercer um papel essencial na assistência ao idoso em cuidados paliativos. Contudo, verifica-se em muitos casos inexperiência da equipe, e baixo envolvimento dos profissionais na assistência paliativa (Andrade, Almeida, Sousa, \& Timm, 2020). Em estudo exploratório envolvendo idosos terminais no contexto de internação hospitalar verificou-se que muitas vezes a autonomia do idoso é violada, prevalecendo a vontade da família ou mesmo o paternalismo profissional. $\mathrm{O}$ estudo ressalta a importância da ampliação dos conceitos e práticas de cuidados paliativos, com respeito à individualidade, valores e sobretudo, protagonismo do idoso nas escolhas que permeiam a terminalidade. Mesmo em casos onde a capacidade cognitiva do idoso esteve preservada, percebeu-se a subjugação deste nas decisões clínico-assistenciais (Gaspar, Silva, Zepeda, \& Silva, 2020). Quanto à compreensão da equipe de saúde sobre o conceito de cuidados paliativos voltado aos idosos verifica-se grande ênfase no alívio da dor e sofrimento.

O envolvimento da família, e abordagem dos aspectos emocionais, culturais e espirituais muitas vezes não são levados em consideração, desde a definição de cuidados paliativos, até no processo de instituição dos mesmos. Um dos grandes desafios, na visão dos profissionais, seria a comunicação com a família sobre a indicação de cuidados paliativos. A aceitação do processo de terminalidade e morte pelos familiares é um processo complexo, mas que pode ser facilitado pela prática de informações continuadas sobre as condições do idoso, deterioração orgânica, e importância da humanização do cuidado e redução do sofrimento (Queiroz, et al., 2018). A atitude do médico frente ao idoso em situação de terminalidade também foi discutido em outro estudo da literatura pesquisada. Um dos estudos problematiza a "mentira terapêutica", ou seja, a atitude muitas vezes paternalista do médico, que numa visão centrada no modelo biomédico omite do paciente sua condição de saúde, bem como sua condição de terminalidade. Ressalta-se a importância do respeito à autonomia do idoso e de seus direitos quanto ao entendimento do seu quadro de saúde, opções terapêuticas existentes, e melhor explicação do processo de cuidados paliativos. O médico precisa ser referência para o paciente na indicação de opções, mas também na transparência de informações clínicas (Macedo, 2020). Seja no contexto hospitalar, ou na assistência em cuidados paliativos domiciliar a equipe de enfermagem é referida como definidora de práticas de cuidado, humanização e acolhimento. Por estarem mais próximos ao leito, e em maior contato com idoso e familiares tais profissionais podem contribuir de forma significativa para melhor enfrentamento da terminalidade, morte e luto. Novamente é ressaltada a importância de proporcionar autonomia ao idoso, para que o mesmo seja "senhor da sua história", garantindo respeito aos seus valores, cultura, e preferências nos momentos finais de vida (Lindolpho, Caldas, Sá, \& Santos, 2016).

\section{CONCLUSÃO}

Evidenciou-se a existência de uma assistência em cuidados paliativos extremamente deficiente, sobretudo quando esta assistência é voltada ao idoso. Há despreparo dos serviços assistenciais, gestão em saúde e também dos profissionais para prestar os cuidados necessários e concomitantemente respeitar valores, cultura e preferências do idoso. A assistência centrada no modelo biomédico, com paternalismo médico, e deficiência de comunicação entre profissionais e idoso foi um tema recorrente nos estudos. Em muitos casos verifica-se desrespeito à vontade e autonomia do idoso, com prevalência de decisões médicas, e/ou decisões familiares, que não condizem às preferências do idoso. Quanto ao local de assistência à pacientes idosos em cuidados paliativos verifica-se que muitos pacientes permanecem em UTIs por longos meses e anos, não sendo de fato instituídos cuidados paliativos adequados. 
Quanto em regime domiciliar fica latente ainda o despreparo da equipe e da rede de saúde para assistência adequada.

\section{REFERÊNCIAS}

Abenante, E. 2021. Centenário da senescência. Rev. Longeviver, 3(10), 43-51.

Academia Nacional de Cuidados Paliativos. 2009. Manual de Cuidados Paliativos. Rio de Janeiro: Diagraphic.

Alves, R., Cunha, E., Santos, G., \& Melo, M. 2019. Cuidados Paliativos: Alternativa para o Cuidado Essencial no Fim da Vida. Psicol. cienc. prof., 39(1), e185734.

Andrade, D., Almeida, M., Sousa, F., \& Timm, M. 2020. O papel da atenção primária à saúde em cuidados paliativos de idosos. Braz. J. of Develop, 6(6), 35307-35320.

Arcanjo, S., Saporetti, L., Curiati, J., Jacob-Filho, W., \& Silva, T. 2018. Características clínicas e laboratoriais associadas à indicação de cuidados paliativos em idosos hospitalizados. Einstein, 16(1), eAO4092.

Ciosak, S., Braz, E., Costa, M., Nakano, N., Rodrigues, J., Alencar, R., \& Rocha, A. 2011. Senescência e senilidade: novo paradigma na atenção básica de saúde. Rev. esc. enferm. USP, 1763-1768.

Clara, M., Silva, V., Alves, R., \& Coelho, M. 2019. Escala Palliative Care Screening Tool como instrumento para indicação de cuidados paliativos em idosos. Rev. Bras. Geriatr. Gerontol, 22(5), e190143.

Correia, S., Nascimento, A., Santos, B., Santos, L., \& Oliveira, C. 2017. Importância do conhecimento em cuidados paliativos na formação dos acadêmicos de Enfermagem: Revisão Integrativa. INTERNATIONAL NURSING CONGRESS (pp. 9-12). Garanhuns - PE: Universidade Tiradentes - UNIT.

Farias, R., \& Santos, S. (2012). Influência dos determinantes do envelhecimento ativo entre idosos mais idosos. Texto contexto enferm., 21(1), 167-176.
Gaspar, R., Silva, M., Zepeda, K., \& Silva, Í. 2020. Conditioning factors for nurses to defend the autonomy of the elderly on the terminality of life. Rev. bras. enferm, 73(3), e20180857.

Gomes, A., \& Othero, M. (2016). Cuidados Paliativos. Estud. av., 30(88), 155-156.

Lindolpho, M., Caldas, C., Sá, S., \& Santos, N. 2016. Cuidados de enfermagem ao idoso no fim da vida. Ciênc. cuid. saúde, 15(2), 383-389.

Liposcki, S. 2016. Influência de um programa de exercícios do método pilates sobre a massa óssea de idosas sedentárias. Pontifícia Universidade Católica do Rio Grande do Sul, Instituto de Geriatria e Gerontologia. Porto Alegre: Programa de PósGraduação em Gerontologia Biomédica.

Macedo, J. (2020). A mentira terapêutica e o silenciamento do idoso e do morrer. Sex., salud soc., 1(35), 237-259.

Marques, F., \& Bulgarelli, A. 2020. Os sentidos da atenção domiciliar no cuidado ao idoso na finitude: a perspectiva humana do profissional do SUS. Ciênc. Saúde Colet, 25(6), 2063-2072.

Miranda, G., Mendes, A., \& Silva, A. 2016. O envelhecimento populacional brasileiro: desafios e consequências sociais atuais e futuras. Rev. bras. geriatr. gerontol., 19(3), 507-519.

Queiroz, T., Ribeiro, A., Guedes, M., Coutinho, D., Galiza, F., \& Freitas, M. 2018. Cuidados paliativos ao idoso na terapia intensiva: olhar da equipe de enfermagem. Texto contexto enferm., 27(1), e1420016.

Ribeiro, A. 2017. Prática de cuidados paliativos em idosos com demência. Universidade de Brasília, Centro de Estudos avançados multidisciplinares. Brasília: Especialização em Saúde da Pessoa Idosa.

Tavares, M., \& Lima, C. 2017. Dificuldades do idoso e familiares na medicação domiciliar. Rev. Interfaces, 4(12), 23-31.

WHO, W. 2002. National cancer control programmes: policies and managerial guidelines. Geneva, Suiça: WHO.

World Health Organization - WHO. (22 de Março de 2017). Palliative Care. Fonte: World Health Organization: https://www.who.int/health-topics/palliative-care 